\title{
Optimal spatial allocating of wind turbines taking externalities into account
}

\author{
Jürgen Meyerhoff ${ }^{1}$, Martin Drechsler ${ }^{2, *}$ \\ ${ }^{1}$ Technische Universität Berlin, Berlin,Germany Germany \\ ${ }^{2}$ UFZ - Helmholtz Centre for Environmental Research, Leipzig, Germany \\ *Corresponding author. Tel: +49 3412351713, Fax: +49 3412351473, E-mail: martin.drechsler@ufz.de
}

\begin{abstract}
Wind power is one of the most promising options for producing energy in a climate-friendly manner. However, besides its environmental benefits wind power generation causes externalities such as impacts on humans and biodiversity. All studies conducted so far show that these externalities can be substantial. The question is how this knowledge translates into a welfare-optimal spatial allocation of turbines that needs to consider both production and external costs. We present a modeling approach for the determination of the welfare-optimal spatial allocation of wind turbines (WT) and apply it to the planning region West Saxony in Germany. The approach combines choice experiments, a non-market valuation method used to measure externalities of wind power, and spatially explicit ecological-economic modeling within an optimization framework. Optimal is understood here as producing a given amount of wind power at lowest social costs. Social costs comprise (i) externalities measured by the (monetized) impact of WT on biodiversity, the distance of the WT to settlements, the height of the WT and size of wind farms, and (ii) the construction and operating costs associated with the WT. We show that the social costs of wind power production can be reduced substantially if externalities are taking into account.
\end{abstract}

Keywords: choice experiment, externality, modeling, spatial allocation, welfare-optimal, wind power.

\section{Introduction}

Wind power belongs to the most efficient renewable energy sources and constitutes an important component of the energy mix in many countries. In future, wind power is going to expand further to help meeting ambitious energy and climate policy goals. However, despite its doubtless advantages, wind power generation comes along with considerable negative externalities that lead to conflicts with other important policy goals, including human health and biodiversity conservation. Human health is affected because of the shade and noise effects produced by wind turbines (WT) [1]. Visual impacts of WT on landscapes have been considered by $[2,3]$. Biodiversity is affected especially through increased mortality and habitat loss for birds and bats [4,5]. External costs of wind power have been quantified, e.g., by [6-8]; see [9] for an overview.

The quality and extent of the monetary and non-monetary externalities of wind power considerably depend on the characteristics of the sites selected for wind power development. One the one hand the unit cost of wind-generated electricity depends on the energy produced per year and this depends on the local wind conditions. On the other hand, WT erected in the vicinity of settlements or bird habitats increase the impact on humans and birds. Different sites available for the installation of a WT will have different pros and cons in terms of wind power production costs and external costs. Since the pros and cons of wind power generation vary in space, they can be balanced and conflicts with other policy goals be mitigated through the appropriate spatial allocation of the WT. In the present article we propose a welfareeconomic approach that determines the spatial allocation in a way that the social cost of producing a given amount of wind power is minimized. The mentioned balance of the pros and cons is reflected by the fact that the social cost is calculated as the sum of the production cost (e.g., installation and operating costs) and the external costs. The latter comprise the 
monetized impacts on human health, biodiversity, etc. and depend on the preferences of the people, i.e. on how they value these impacts.

Despite their advantages such as their analytical clarity and rootedness in economic theory, welfare-economic approaches have been rarely used to optimize the spatial allocation of land use in general, and WT in particular. An example that goes into this direction is found in [10] which explores trade-offs between wind power production and the conservation of two animal species. However, since the analysis does not include any information about social preferences, it cannot determine the welfare-optimal allocation of WT. The above-mentioned studies [6-9] on the other hand, provide the necessary information about social preferences but do not include any assessment or modeling of how the valued impacts depend on the spatial allocation of the WT. As a consequence, they too cannot determine the welfareoptimal spatial allocation. This lack has recently been criticized [11]. The two approaches, spatially explicit modeling of impacts and the economic valuation of impacts have complementary strengths and weaknesses and combining them allows overcoming the weaknesses. In the present paper we follow this route to determine the welfare-optimal spatial allocation of WT in a study region in Germany. For this region we investigate a number of policy relevant questions: (i) how do society's preferences affect the welfare-optimal allocation of WT, (ii) what is the trade-off between the production and the external costs of wind power production, and (iii) what are the consequences of ignoring external cost in the planning of landscapes for wind power production.

The paper is structured as follows. In section 2 we will outline the modeling approach and present the study region. In section 3 we apply the modeling approach to the study region and present the results in section 4 . Section 5 discusses the results and draws conclusions for policy design.

\section{Methodology}

\subsection{The modeling approach}

The objective of the analysis is to allocate WT in the study region such that a given level of electricity $E_{\min }$ is produced per year at minimal social cost $C$. The social cost of wind power supply is composed of the production costs $C_{p}$ and external costs $C_{e}$. To determine external costs we define attributes that capture the relevant externalities as identified through stakeholder interviews (see section 3.1 below). The attributes are quantified through spatially explicit models and valued through choice experiments. In the present case the attributes comprise: the loss rate $(L)$ of important species, the minimum distance of WT to settlements $(D)$, the height of the installed WT $(H)$ and the size of wind parks $(S)$. The attributes $D, H$ and $S$ consider the impact of WT on the landscape and ultimately human inhabitants. The disturbance of humans by the noise of a WT depends, among others, on the height of the WT and its distance to the settlement areas. Attribute $H$ considers that WT technologies with different heights may by installed. Attribute $S$ considers that WT may be allocated in larger or smaller wind parks. The production cost and attribute $L$ depend on the time frame. We consider a time frame of 20 years, which is about the life time of a WT, so $C_{p}$ measures production costs over 20 years and $L$ measures species decline within 20 years. The analysis is carried out in several steps. First we construct the social cost function

$$
C=C_{p}+C_{e}(L, D, H, S)
$$


where $C_{e}$ are the external costs associated with the attributes $L, D, H$ and $S$. They are determined through choice experiments (see section 3.1). We further identify the sites that are physically and legally suitable for the installation of a WT. Given these potential sites, WT allocation strategies are formed as described above, considering that the energy target $E_{\text {min }}$ must be fulfilled. For each allocation strategy we determine the associated attributes $C_{p}, L, D$, $H$ and $S$ and determine the social cost $C$. For given energy target $E_{\min }$, the welfare-optimal allocation of WT, i.e. the allocation that minimizes $C$, is determined through numerical optimization.

\subsection{Application of the modeling approach}

The approach is applied to the Planning Region West Saxony in Germany that is a part of the Free State of Saxony. The region has about 500,000 households (2005) and covers an area of around $4,300 \mathrm{~km}^{2}$. Due to its topography the region is fairly suited for wind power production but at the same time belongs to the core distributional area of the endangered Red Kite (Milvus milvus). Red Kites have been frequently observed to be killed by WT. The Red Kite therefore forms the focal bird species in our analysis and $L$ measures the rate by which the Red Kite population declines as a consequences of the presence of WT in the region [12]. Below we go through the steps of the modeling approach.

\subsubsection{Construction of the external cost function through choice experiments}

We consider an external cost function which is the sum of the partial external costs $C_{y}(y)$ associated with the attributes $y \in\{L, D, H, S\}$ :

$$
C_{e}(L, D, H, S)=\sum_{y \in\{L, D, H, S\}} C_{y}(y) \cdot \sum_{t=1}^{T}(1+r)^{-t}
$$

The partial external cost $C_{y}(y)$ represents the cost for a single year. Since we are considering a time span of $T=20$ years, we have to aggregate the costs over these 20 years. We discount the external costs at annual rate $r$. We assume that $C_{y}(y)$ has the shape $C_{y}(y)=a_{y} /\left(y-b_{y}\right)+g_{y}$ (which can describe concave or convex increases or decreases of $C_{y}$ with $y$ ) and carry out choice experiments (CE) [13] to determine the parameters $a_{y}, b_{y}$ and $g_{y}$ for all $y \in\{L, D, H, S\}$. CE are based on the assumption that the utility to consumers of any good (i.e., also public goods such as a landscape) is derived from its attributes or characteristics. Due to this focus CE are particularly useful for valuing multidimensional changes. In a CE, respondents are asked to make comparisons among environmental alternatives characterized by a variety of attributes and the levels of these. Typically, respondents are offered multiple choices during the survey, each presenting alternative designs of the environmental change in question and the option to choose the status quo. The record of choices serves as a basis to estimate the respondents' willingness to pay. Changes in welfare due to a marginal change in a given attribute are calculated using the MWTP. It is defined as the maximum amount of income a person will pay in exchange for an improvement in the level of a given attribute provided and can be identified as the difference in $C_{y}$ associated with the change in $y$.

In the present study we consider the four different attributes $L, D, H$ and $S$ with three levels for each attribute to characterize changes in the environment. They are combined to choice sets using an experimental design that allows determination of the independent influence of the attributes on respondents' choices (details of the CE can be found in [9]). The choices of 353 randomly chosen inhabitants from the study region were considered. 


\subsubsection{Specifying the decision space and modeling the attributes}

We start our analysis by identifying which parts in the landscape are physically and legally qualified for the allocation of WT with the help of a geographical information system (GIS) of the region. Broadly speaking, these are open areas distant enough from infrastructure, settlements and nature conservation areas. The analysis focuses on two WT technologies $k=1,2$. The $k=1$ type has a hub height of $78 \mathrm{~m}$ and rotor diameter of $82 \mathrm{~m}$, yielding a nominal power of $2 \mathrm{MW}$, while the $k=2$ type has a hub height of $105 \mathrm{~m}$ and a rotor diameter of $90 \mathrm{~m}$, yielding a nominal power of 3MW. The suitable parts of the landscape are subsequently filled with a grid of points with each point in the grid representing a potential site for the allocation of a WT, taking technical minimum distances between individual WT into account. The number of potential sites is $N=1098$. Allocation scenarios are defined by deciding for each potential WT site $i=1, \ldots, N$ whether it should contain a WT of type 1 or type 2 or no WT.

The energy yield $E_{i k}$ for each site $i$ and WT type $k$ is calculated by using the technical parameters of the WT and the relevant frequency distribution of wind speeds observed at the spatial location and altitude of the WT hub (for further details see [12]). The wind speed data were obtained from Eurowind GmbH (Köln, Germany). The total energy $E_{\text {tot }}$ produced per year in the region is obtained by summing $E_{i k}$ over all installed WT.

The production cost $C_{p, k}$ (over a time frame of 20 years) associated with a WT of type $k$ comprises the construction and operating costs. The construction costs are composed of selling prices, taken from the companies' price lists, and a 10 percent mark-up to cover on-site construction costs, including grid connection. Annual operating costs are typically estimated at five percent of the construction costs (information provided by interviewed WT operators).

Ecological externalities are partly taken into account by prohibiting the erection of WT in areas protected by nature conservation laws. However, protected areas are by no means sufficient to reach the ambitious goals of biodiversity policy. So the impacts of WT on biodiversity have to be considered even if the WT are installed outside the protected areas. We consider $L$, the percentage of the regional Red Kite population that is lost due to WT over the modeling time frame of 20 years. We model this loss rate as the sum of "marginal" loss rates $l_{i}$ over all sites $i=1, \ldots, N$ that contain a WT. We assume that the contribution $l_{i}$ of site $i$ to $L$ is determined by the probability of an individual of the focal species being found at the site. This depends, e.g., on how close the site is to a nest, whether the site is located within a migratory bird route, etc. In the case of Red Kites we assume that $l_{i}$ is a declining function of the distances of site $i$ to known nests (i.e., WT close to a nest cause a higher collision risk for the Red Kite with the WT than WT at more distance to a nest).

The modeling of the remaining three attributes is straightforward. Attribute $D$ represents the minimum distance of WT to settlements, considering all settlements and installed WT in the region. Attribute $H$ is modeled as the average over the heights of all installed WT in the region. To determine attribute $S$ we apply the wind park method [14] that clusters all WT into wind parks. $S$ is then the average size of those wind parks. 


\section{Results}

\subsection{Construction of the social cost function}

The choice experiments yield the following MWTP (in Euros per household per month) ${ }^{1}$ : 2.13 for an improvement in the Red Kite loss rate $L$ from $10 \%$ to $5 \%$ per 20 years; -2.18 for a worsening in $L$ from $10 \%$ to $15 \%$; 3.18 for an increase in the settlement distance $D$ from $800 \mathrm{~m}$ to $1100 \mathrm{~m}$; and 3.94 for an increase in $D$ from $800 \mathrm{~m}$ to $1500 \mathrm{~m}$. Changes in the other two attributes, $H$ and S, did not lead to a statistically significant MWTP.

The partial cost functions $C_{y}(y)$ (eq. 2) are fitted to these measured MWTP to obtain the external cost function $C_{e}(L, D)$ shown in Fig. 1. One can see that external costs increase with increasing externalities, i.e. with increasing Red Kite loss rate $L$ and decreasing settlement distance $D$.

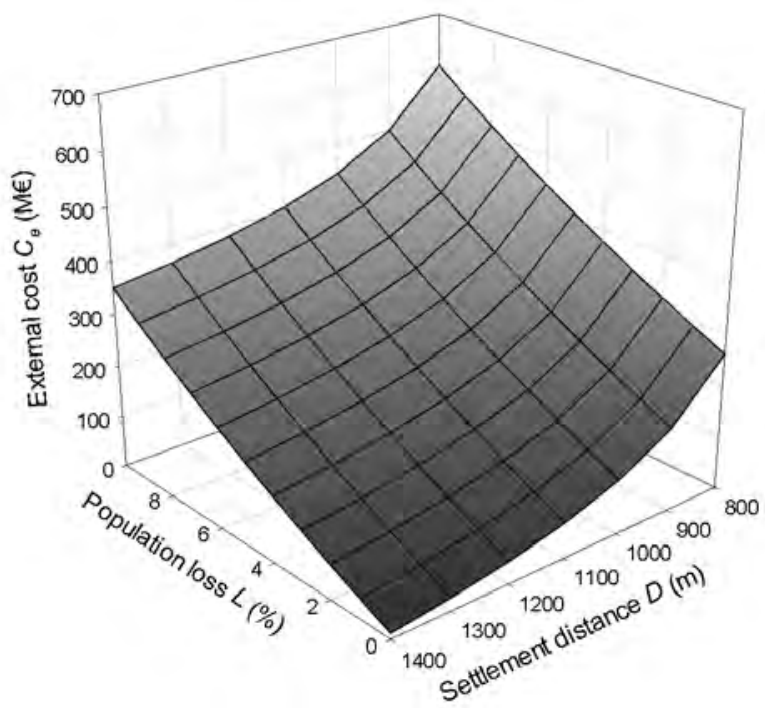

Fig. 1. External cost $C_{e}=C_{L}+C_{D}$ for the study region as a function of Red Kite loss rate $L$ and settlement distance $D$, considering a time frame of 20 years with annual discount rate $r=3 \%$.

\subsection{Evaluation of the attributes}

Figure 2a shows the production cost $C_{p}$ (over 20 years, discounted to the present year) for all potential WT sites $i$ in the region. One can see that the production costs are lowest in the south, centre and east, and highest in the north east, which reflects relatively high wind speeds and large energy outputs $E_{i k}$ in the south, centre and east and low wind speeds and energy outputs in the north east.

The external costs are determined by the settlement distance $D$ and the Red Kite loss rate $L$ which is the sum of the impacts $l_{i}$ associated with each WT site $i$. The impacts for all potential WT sites are shown in Fig. 2b. We find that the $l_{i}$ are relatively uncorrelated to the production costs, so there are both "low-conflict" WT sites that have low (high) production costs and low (high) impact on the Red Kite and "high-conflict" sites with low (high) production costs and high (low) impact on the Red Kite.

\footnotetext{
${ }^{1}$ The conditional logit reveals that only the attributes $L$ and $D$ have a significant influence on respondents' choices among the alternatives presented on the choice sets. Thus, only for these attributes MWTPs are calculated. See [9] for more details on the analysis of the choice experiments.
} 


\subsection{The welfare-optimal allocation of WT}

The task is to allocate WT to the potential sites $i=1, \ldots, N$ so that the energy target $E_{\min }=690$ GWh per year is achieved at minimal social cost where social cost is given by the sum of production costs (cf. Fig. 2a) and external costs (Fig. 1). The resulting optimal WT allocation scenario is characterized by the following welfare-optimal levels of the attributes: the optimal Red Kite population loss rate is $L^{*}=1.2$ percent within 20 years, the optimal settlement distance is $D^{*}=1,025 \mathrm{~m}$ and the optimal production cost amounts to $C_{p}{ }^{*}=730$ million Euros (sum over 20 years, present value, discounted at 3\% per year). Altogether, a number of 122 large WT types but no small WT are installed.

To understand the trade-offs between production and external costs we determined the optimal allocation of WT under the assumption that the MWTP for avoiding externalities are reduced to one tenth of the observed values (cf. section 3.1). By assuming that society places little value to the externalities of the WT ( $L$ and $D$ ) this scenario considers mainly the production costs, and WT are allocated so that the total production cost $\left(C_{p}\right)$ for reaching the energy target $E_{\min }$ are minimized. Consequently, the $690 \mathrm{GWh}$ per year can be produced at a production cost of only $C_{p}{ }^{*}=690$ million Euros. While production costs are reduced, the optimal levels of the externalities are increased in this scenario: $L^{*}$ increases to 2.6 percent within 20 years and $D^{*}$ reduces to $800 \mathrm{~m}$. That means that the price for reducing the production cost is an increase in the external costs. According to Fig.1, increasing $L$ from 1.2 to 2.6 and reducing $D$ from $1025 \mathrm{~m}$ to $800 \mathrm{~m}$ increases the external cost by about 210 million Euros. So altogether, ignoring the externalities saves 40 million Euros of production costs but raises external costs by 210 million Euros and on net raises social cost by 170 million Euros.
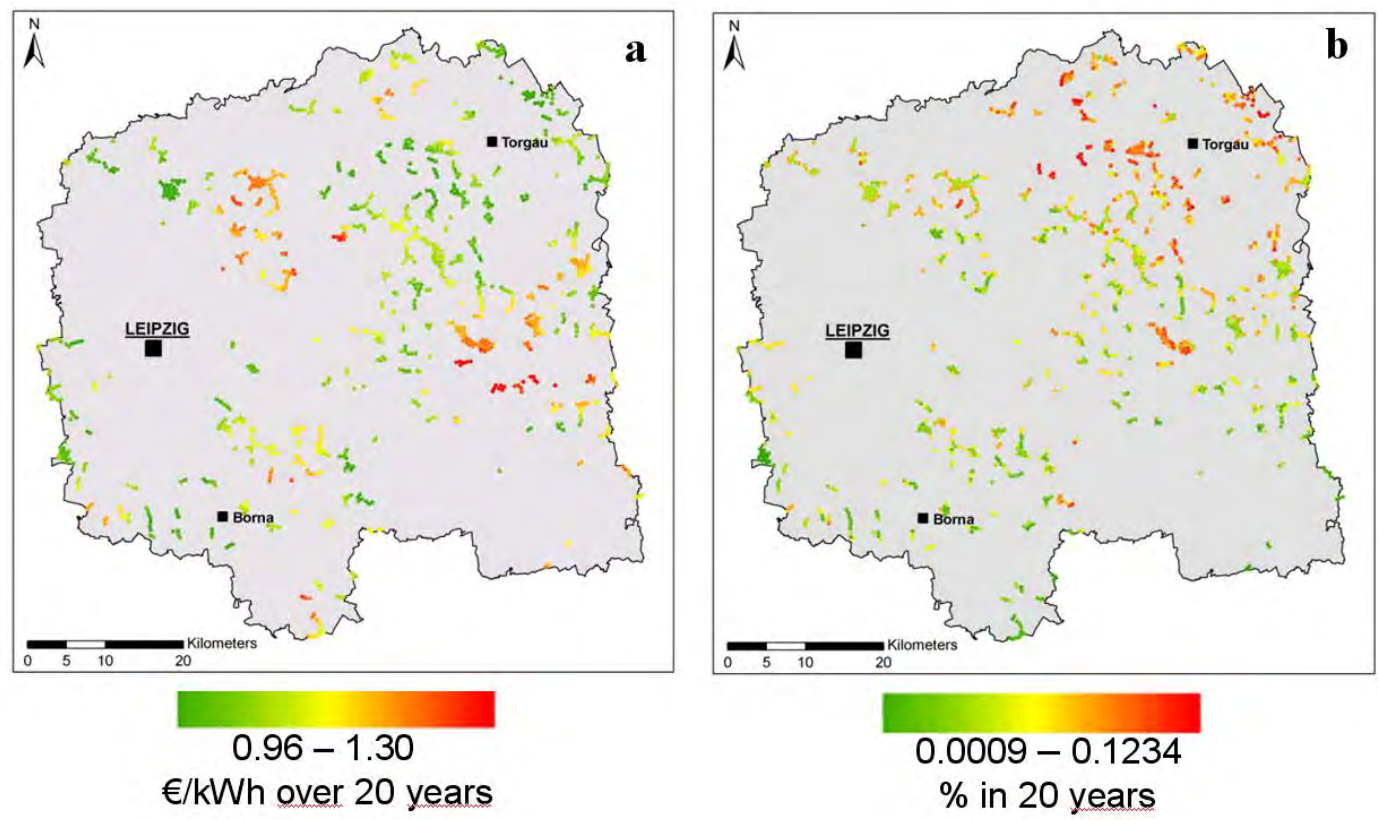

Fig. 2. a: Present value cost of producing $1 \mathrm{kWh}$ over 20 years for each WT site, represented by color scale; $b$ : contribution to Red Kite loss $\left(l_{i}\right.$ : see text) of each potential WT site $i$, represented by color scale. Small WT $(k=1)$ are installed at sites between $750 \mathrm{~m}$ and $1000 \mathrm{~m}$ from settlements, large WT $(k=2)$ at larger distances $>1000 \mathrm{~m}$.

\section{Discussion and Conclusions}

Wind power is one of the most promising options for producing energy in a climate-friendly manner. However, it causes negative externalities in terms of adverse impacts on humans and 
biodiversity. To alleviate the conflict between the positive impact of wind power on climate policy and its negative externalities, wind turbines (WT) should be allocated so that social costs (i.e., the sum of wind power production costs plus external costs) are minimized with regard to the desired climate-friendly energy target. Determining such an allocation ex ante requires the combination of different methods, including economic modeling of the production costs, modeling of the non-monetary external effects, monetary valuation of these external effects, and numerical optimization.

The goal is to reach a certain energy target in a concrete region $\left(E_{\min }\right)$ in a welfare-optimal manner, i.e. at lowest social costs. To do this, we explored trade-offs between production costs and externalities by combining choice experiments and spatially explicit modeling. It turned out that in the study region the distance of WT to settlements and the impact on a focal species, the Red Kite, represent significant externalities of wind power supply. On the other hand wind park size and height of the turbines are not. The welfare-optimal allocation balances production costs and externalities and minimizes the sum of production costs and external costs. When determining the welfare-optimal allocation of WT in our study region in Germany it turned out that ignoring the externalities and minimizing only the production costs would reduce the production costs by about five percent from 730 million Euros to 690 million Euros but increase the external cost by 210 million Euros each compared to the welfare optimum. Altogether, ignoring the externalities would increase social cost by 210$(730-690)=170$ million Euros.

The magnitude of the chosen energy target reflects the importance of producing energy in a climate friendly manner. The optimal magnitude should be chosen such that the regional social costs of wind power production are outweighed by the benefits accruing from reduced CO2 emission. Determining the "globally" optimal level of the energy target, however, was beyond the scope of this study and is a matter of future research.

The numbers obtained in the analysis depend on several assumptions. In the assessment of the impacts of WT on the Red Kite, e.g., we ignored options of on-site management that make sites unattractive for the Red Kite and would thus reduce the modeled collision risk. Moreover, the search range of Red Kites is not circular. Information on the search behavior of Red Kites, however, is difficult to obtain and requires sophisticated field observations. If this information was at hand it could be easily fed into the model.

In the establishment of the external cost function $\left(C_{e}\right.$, eq. (2)) we assumed that it is separable and can be written as the sum of partial costs. This ignores the possibility of interactions so that the marginal willingness to pay for the reduction of one externality depends on the level of another externality. In the choice experiments we searched for such interactions but did not find any significant ones. If they are significant, interactions can be considered by an appropriate adaptation of the shape of $C_{e}$.

With regard to the production costs we ignored the spatial variation of grid connection costs. These very much depend on the distance of a site to the next feed in station, and also on whether a solitary WT or a wind park is connected. Generally connection costs per WT decrease with increasing size of a wind park (economy of scale). Taking these factors into account would require detailed knowledge about the present power grid and even more, assumptions how allocation of WT and expansion of the power grid co-evolve. Since the evolution of power grids and the installation of new smart technologies to make existing 
power grids effective for renewable energies are currently a hot topic it may be interesting to further explore this issue in future.

\section{Acknowledgements}

The authors thank the German Ministry for Education and Research for funding the research project "Conflicts of interest over wind power" [Nachhaltige Landnutzung im Spannungsfeld umweltpolitisch konfligierender Zielsetzungen am Beispiel der Windenergiegewinnung] (Grant No. 01UN0601A, B) as a part of the fona Programme "Economics for Sustainability [Wirtschaftswissenschaften für Nachhaltigkeit] (WIN)". The views expressed in this paper are the responsibility of the authors alone. In addition we thank the West Saxony Regional Planning Office for the constructive cooperation and commentary.

\section{References}

[1] E. Hau, Wind Turbines. Fundamentals, Technology, Application, Economics. Springer, Berlin/Heidelberg, Germany, 2006.

[2] C.L. Krause, Our visual landscape: Managing the landscape under special consideration of visual aspects. Landscape and Urban Planning 54, 2001, pp. 239-254.

[3] B. Möller, Changing wind-power landscapes: regional assessment of visual impact on land use and population in Northern Jutland, Denmark. Applied Energy 83, 2006, pp. 477-494.

[4] H. Hötker, K.M. Thomsen, H. Jeromin, H., Impacts on biodiversity of exploitation of renewable energy sources: the example of birds and bats - facts, gaps in knowledge, demands for further research, and ornithological guidelines for the development of renewable energy exploitation. Michael-Otto-Institut im NABU, Bergenhusen, Germany, 2006

(http://www.batsandwind.org/pdf/impacts\%20on\%20biodiversity\%20of\%20renewable\% 20energy.pdf).

[5] J. Bright, R. Langston, R. Bullman, R., Evans, S. Gardener, J. Pearce-Higgins, Map of bird sensitivities to wind farms in Scotland: A tool to aid planning and conservation, Biological Conservation 141, 2008, pp. 2342-2356.

[6] B. Álvarez-Farizo, N. Hanley, Using conjoint analysis to quantify public preferences over the environmental impacts of wind farms. An example from Spain, Energy Policy 30, 2002, pp. 107-116.

[7] K. Ek, Quantifying the environmental impacts of renewable energy: The case of Swedish wind power, in: D. Pearce D. (Ed.), Environmental Valuation in Developed Countries: Case Studies. Edward Elgar, Cheltenham, 2006, pp. 181-210.

[8] A. Dimitropoulos, A. Kontoleon, Assessing the determinants of local acceptability of wind farm investment: a choice experiment in the Greek Islands. University of Cambridge, UK, Department of Land Economics, Environmental Economy and Policy Research Working Paper No. 35.2008.

[9] J. Meyerhoff, C. Ohl, V. Hartje, Landscape externalities from onshore wind power. Energy Policy 38, 2010, pp. 82-92.

[10]M.J. Punt, R.A. Groeneveld, E.C. van Ierland, J.H. Stel, Spatial planning of offshore wind farms: a windfall to marine environmental protection? Ecological Economics 69, 2009, pp. 93-103. 
[11]J. Ladenburg, Onshore and offshore locations for wind power development - what does the public prefer and should it matter? Modern Energy Review 1, 2009, pp. 32-34.

[12]M. Eichhorn, M. Drechsler, Spatial trade-offs between wind power production and bird collision avoidance in agricultural landscapes, Ecology and Society 15, 2010 [online] URL: http://www.ecologyandsociety.org/vol15/iss2/art10/.

[13]J.J. Louviere, D.A. Hensher, J.D. Swait, Stated Choice Methods. Analysis and Application. Cambgridge University Press. Cambridge, 2000.

[14]M. Schmitt, F. Dosch, E. Bergmann, Flächeninanspruchnahme durch Windkraftanlagen. Berichte aus Forschung und Praxis. Raumforschung und Raumordnung 64/5, 2006, pp. 405-412. 